\title{
Joint Distribution of Eigenvalues of Linear Stochastic Systems
}

\author{
Sondipon Adhikari $^{*}$ \\ University of Bristol, Bristol, United Kingdom
}

\begin{abstract}
Description of real-life engineering structural systems is associated with some amount of uncertainty in specifying material properties, geometric parameters, boundary conditions and applied loads. In the context of structural dynamics it is necessary to consider random eigenvalue problems in order to account for these uncertainties. Current methods to deal with such problems are dominated by approximate perturbation methods. Some exact methods to obtain joint distribution of the natural frequencies are reviewed and their applicability in the context of real-life engineering problems are discussed. A new approach based on an asymptotic approximation of multidimensional integrals is proposed. A closedform expression for general order joint moments of arbitrary number of natural frequencies of linear stochastic systems is derived. The proposed method does not employ the 'small randomness' assumption unusually used in perturbation based methods. Joint distributions of the natural frequencies are investigated using numerical examples and the results are compared with Monte Carlo Simulation.
\end{abstract}

\section{Introduction}

ChARACTERIZATION of the natural frequencies and the mode-shapes play a fundamental role in the analysis $\bigcup$ and design of engineering dynamic systems. The determination of natural frequency and mode shapes require the solution of an eigenvalue problem. Eigenvalue problems also arise in the context of the stability analysis of structures. This problem could either be a differential eigenvalue problem or a matrix eigenvalue problem, depending on whether a continuous model or a discrete model is used to describe the given vibrating system. Description of real-life engineering structural systems is inevitably associated with some amount of uncertainty in specifying material properties, geometric parameters, boundary conditions and applied loads. When we take account of these uncertainties, it is necessary to consider random eigenvalue problems. Several studies have been conducted on this topic since the mid-sixties. The study of probabilistic characterization of the eigensolutions of random matrix and differential operators is now an important research topic in the field of stochastic structural mechanics. The paper by Boyce ${ }^{1}$ and the book by Scheidt and Purkert ${ }^{2}$ are useful sources of information on early work in this area of research and also provide a systematic account of different approaches to random eigenvalue problems. Several review papers, for example, by Ibrahim, ${ }^{3}$ Benaroya and Rehak, ${ }^{4}$ Benaroya, ${ }^{5}$ Manohar and Ibrahim, ${ }^{6}$ and Manohar and Gupta ${ }^{7}$ have appeared in this field which summarize the current as well as the earlier works.

In this paper we obtain a closed-form expression of arbitrary order joint moments of the natural frequencies of discrete linear systems or discretized continuous systems. The random eigenvalue problem of undamped or proportionally damped systems can be expressed by

$$
\mathbf{K}(\mathbf{x}) \phi_{j}=\omega_{j}^{2} \mathbf{M}(\mathbf{x}) \phi_{j}
$$

Here $\omega_{j}$ and $\phi_{j}$ are the natural frequencies and mode shapes of the dynamic system. $\mathbf{M}(\mathbf{x}): \mathbb{R}^{m} \mapsto \mathbb{R}^{N \times N}$ and $\mathbf{K}(\mathbf{x}): \mathbb{R}^{m} \mapsto \mathbb{R}^{N \times N}$, the mass and stiffness matrices, are assumed to be smooth, continuous and at

${ }^{*}$ Lecturer, Department of Aerospace Engineering, University of Bristol Queens Building, University Walk, Bristol BS8 1TR, UK, AIAA Member.

Copyright (c) 2005 by the American Institute of Aeronautics and Astronautics, Inc. The U.S. Government has a royalty-free license to exercise all rights under the copyright claimed herein for Governmental purposes. All other rights are reserved by the copyright owner. 
least twice differentiable functions of a random parameter vector $\mathbf{x} \in \mathbb{R}^{m}$. The vector $\mathbf{x}$ may consist of material properties, e.g., mass density, Poisson's ratio, Young's modulus; geometric properties, e.g., length, thickness, and boundary conditions. Statistical properties of the system are completely described by the joint probability density function $p_{\mathbf{x}}(\mathbf{x}): \mathbb{R}^{m} \mapsto \mathbb{R}$. For mathematical convenience we express

$$
p_{\mathbf{x}}(\mathbf{x})=\exp \{-L(\mathbf{x})\}
$$

where $-L(\mathbf{x})$ is often known as the log-likelihood function. For example, if $\mathbf{x}$ is a $m$-dimensional multivariate Gaussian random vector with mean $\boldsymbol{\mu} \in \mathbb{R}^{m}$ and covariance matrix $\boldsymbol{\Sigma} \in \mathbb{R}^{m \times m}$ then

$$
L(\mathbf{x})=\frac{m}{2} \ln (2 \pi)+\frac{1}{2} \ln \|\boldsymbol{\Sigma}\|+\frac{1}{2}(\mathbf{x}-\boldsymbol{\mu})^{T} \boldsymbol{\Sigma}^{-1}(\mathbf{x}-\boldsymbol{\mu})
$$

It is assumed that in general the random parameters are non-Gaussian and correlated, i.e., $L(\mathbf{x})$ can have any general form provided it is a smooth, continuous and at least twice differentiable function. It is further assumed that $\mathbf{M}$ and $\mathbf{K}$ are symmetric and positive definite random matrices so that all the eigenvalues are real and positive.

The aim of studying random eigenvalue problems is to obtain the joint probability density function of the eigenvalues and the eigenvectors. The current literature on random eigenvalue problems arising in engineering systems is dominated by the perturbation methods. These methods work well when the uncertainties are small and the parameter distribution is Gaussian. Methods which are not based on perturbation approach but still have the generality and computational efficiency to be applicable for engineering dynamic systems are rare. Grigoriu ${ }^{8}$ has examined the roots of characteristic polynomials of real symmetric random matrices using distribution of zeros of random polynomials. Lee and Singh ${ }^{9}$ have proposed a direct matrix product (Kronecker product) method to obtain the first two moments of the eigenvalues of discrete linear systems. Nair and Keane ${ }^{10}$ have proposed a stochastic reduced basis approximation which can be applied to discrete or discretized continuous dynamic systems. Recently Adhikari and Langley ${ }^{11}$ and Adhikari and Friswell ${ }^{12}$ have proposed some non-perturbative methods to obtain moments and pdf of the eigenvalues for the general case.

For dynamic response analysis and in many other practical problems joint distribution of the eigenvalues are required. Under very special circumstances, for example when the system matrix is GUE (Gaussian unitary ensemble) or GOE (Gaussian orthogonal ensemble), an exact closed-form expression can be obtained for the joint pdf of the eigenvalues. ${ }^{13}$ However, unfortunately the system matrices of real structures may not always follow such distributions and consequently some kind of approximate analysis is required for structural engineering problems. While several papers are available on the distribution of individual eigenvalues, joint distributions of the eigenvalues seem to have received little attention in literature. To the best of authors knowledge only first-order perturbation results ${ }^{14}$ are available for the joint pdf of the eigenvalues. In this paper few methods are proposed to obtain joint distribution of the natural frequencies of discrete linear systems. In section II some exact results available for the joint probability density function of the eigenvalues are presented and their limitations in the context of engineering dynamic systems have been discussed. In section III first and second order perturbation methods are discussed and the joint statistics of the natural frequencies are derived for the special case when the basic random variables are Gaussian. When the basic random variables are non-Gaussian, a new method to obtain arbitrary order moments of the natural frequencies is proposed in section IV. In section V the proposed analytical methods are applied to a three degree-of-freedom system and the results are compared with Monte Carlo simulations.

\section{Exact Joint Probability Density Function of the Natural Frequencies}

Without any loss of generality the eigenvalue problem in Eq. (1) can be expressed by

$$
\mathbf{H}(\mathbf{x}) \boldsymbol{\psi}_{j}=\omega_{j}^{2} \boldsymbol{\psi}_{j}
$$

where

$$
\begin{array}{ll} 
& \mathbf{H}(\mathbf{x})=\mathbf{M}^{-1 / 2}(\mathbf{x}) \mathbf{K}(\mathbf{x}) \mathbf{M}^{-1 / 2}(\mathbf{x}) \in \mathbb{R}^{N \times N} \\
\text { and } \quad & \boldsymbol{\psi}_{j}=\mathbf{M}^{1 / 2} \boldsymbol{\phi}_{j}
\end{array}
$$


Since $\mathbf{M}$ and $\mathbf{K}$ are assumed to be real, symmetric and positive definite, the matrix $\mathbf{H}$ (the dependence on $\mathbf{x}$ will be omitted for notational convenience) given in Eq. (5) is also a real, symmetric and positive definite matrix. Therefore, its eigenvalues are positive and the matrix of eigenvectors

$$
\boldsymbol{\Psi}=\left[\boldsymbol{\psi}_{1}, \boldsymbol{\psi}_{2}, \cdots, \boldsymbol{\psi}_{N}\right] \in \mathbb{R}^{N \times N}
$$

can be normalized into an orthogonal matrix. The matrix $\boldsymbol{\Psi}$ therefore belongs to the group of $N \times N$ orthogonal matrices, denoted by $O(N)$. For convenience, we arrange the natural frequencies in an ascending order and construct a diagonal matrix containing the natural frequencies as

$$
\boldsymbol{\Omega}=\operatorname{diag}\left[\omega_{1}, \omega_{2}, \cdots, \omega_{N}\right] \quad \text { where } \quad 0<\omega_{1}<\omega_{2} \cdots<\omega_{N}
$$

Using $\boldsymbol{\Psi}$, the system matrix can be transformed to $\boldsymbol{\Omega}$ as

$$
\mathbf{H}=\Psi \boldsymbol{\Omega}^{2} \boldsymbol{\Psi}^{T}
$$

From the probability density function of $\mathbf{x}$ it is possible to obtain the probability density function of $\mathbf{H}$, denoted by $p_{\mathbf{H}}(\mathbf{H})$. Since it is assumed that the natural frequencies are distinct, the probability of occurring $\omega_{i}=\omega_{j}$ for $i \neq j$ is zero. Under these fairly general and physically relevant assumptions, the joint pdf of the natural frequencies can be obtained using Muirhead ${ }^{15}$ (Theorem 3.2.17). The exact joint probability density function of all the $N$ natural frequencies of the system is described in the following theorem:

Theorem 1 The joint probability density function of the natural frequencies of an $N$-dimensional linear positive definite dynamic system is given by

$$
p_{\boldsymbol{\Omega}}\left(\omega_{1}, \omega_{2}, \cdots, \omega_{N}\right)=\frac{\pi^{N^{2} / 2}}{\Gamma(N / 2)} \prod_{i<j \leq N}\left(\omega_{j}^{2}-\omega_{i}^{2}\right) \int_{O(N)} p_{\mathbf{H}}\left(\boldsymbol{\Psi} \boldsymbol{\Omega}^{2} \boldsymbol{\Psi}^{T}\right)(d \boldsymbol{\Psi})
$$

where $\mathbf{H}=\mathbf{M}^{-1 / 2} \mathbf{K} \mathbf{M}^{-1 / 2}$ and $p_{\mathbf{H}}(\mathbf{H})$ is the pdf of $\mathbf{H}$.

By considering the Jacobian of the transformation in Eq. (9), the theorem can be proved following the procedure described by Muirhead. ${ }^{15}$ In the product term in Eq. (10), $i$ varies from 1 to $N$ and for each value of $i, j$ varies from $i+1$ to $N$ so that there are total $N(N-1) / 2$ terms. The integral in Eq. (10) needs to be evaluated over the space of $N \times N$ orthogonal matrices. The probability density function of system the matrix $\mathbf{H}$ in Theorem 1 can be any general smooth continuous density function and it is not restricted to the Gaussian density function. Although Theorem 1 gives the exact closed-form joint pdf of all the natural frequencies of a linear stochastic system with general non-Gaussian parameter distribution, the following difficulties may be encountered for real-life engineering systems:

- the multidimensional integral over the orthogonal group $O(N)$ is difficult to carry out in practice and exact closed-form results can be derived only for few special cases;

- the derivation of an expression of the joint pdf of the system matrix $p_{\mathbf{H}}(\mathbf{H})$ is non-trivial even if the joint pdf of the random system parameters $\mathbf{x}$ is known;

- even one can overcome the previous two problems, the joint pdf of the natural frequencies given by Eq. (10) is 'too much information' to be useful for practical problems because

- it is not easy to 'visualize' the joint pdf in the space of $N$ natural frequencies, and

- the derivation of the marginal density functions of the natural frequencies from Eq. (10) is not straightforward, especially when $N$ is large.

In spite of these difficulties, an expression of joint pdf of the natural frequencies can be useful in many situations, for example, to verify the accuracy of an approximate numerical method. Here we list some special cases for which the integral in Eq. (10) can be carried out analytically and simple closed-form expressions can be obtained for the joint pdf of the natural frequencies. 
- System matrix $\mathbf{H}$ is from a Gaussian orthogonal ensemble (GOE): This case forms the basis of random matrix theory ${ }^{13}$ (RMT) and it has been used extensively in the literature of different subjects, for example on the study of excitation spectra of various atomic nuclei ${ }^{16}$ and high frequency structural vibration. ${ }^{17,18}$ With GOE assumption the ensemble is invariant under every orthogonal transformation

$$
\mathbf{H} \longrightarrow \Psi^{T} \mathbf{H} \Psi
$$

and various elements of $\mathbf{H}_{i j}, i \leq j$ are statistically independent and Gaussian. The pdf of $\mathbf{H}$ can be expressed as

$$
p_{\mathbf{H}}(\mathbf{H})=\exp \left(-\theta_{2} \operatorname{Trace}\left(\mathbf{H}^{2}\right)+\theta_{1} \operatorname{Trace}(\mathbf{H})+\theta_{0}\right)
$$

where $\theta_{2}$ is real and positive and $\theta_{1}$ and $\theta_{0}$ are real. If this two assumptions are satisfied, then the joint pdf of the natural frequencies can be obtained (see Mehta, ${ }^{13}$ Chapter 3 ) as

$$
p_{\boldsymbol{\Omega}}\left(\omega_{1}, \omega_{2}, \cdots, \omega_{N}\right)=\exp \left[-\left(\sum_{j=1}^{N} \theta_{2} \omega_{j}^{4}-\theta_{1} \omega_{j}^{2}-\theta_{0}\right)\right] \prod_{i<j}\left|\omega_{j}^{2}-\omega_{i}^{2}\right|
$$

Although the system matrices of a real-life structural system may not exactly follow the assumption of GOE, the distribution of the spacing between the natural frequencies is very close to that of a GOE matrix. ${ }^{19}$

- System matrix $\mathbf{H}$ has Wishart distribution: If $\mathbf{Z} \in \mathbb{R}^{N \times N}$ is a Gaussian random matirx ${ }^{20}$ with zero mean and covariance matrix $\left[\mathbf{I}_{N} \otimes \mathbf{\Sigma}\right] \in \mathbb{R}^{N^{2} \times N^{2}}$, then the matrix $\mathbf{W}=\mathbf{Z}^{T} \mathbf{Z}$ is said to have a Wishart distribution, commonly denoted as, $W_{N}(N, \Sigma)$. The spectrum of $\mathbf{H}$ in Eq. (5) is exactly same as that of $\mathbf{M}^{-1} \mathbf{K}$. Therefore, if the elements of $\mathbf{M}^{-1}$ and $\mathbf{K}$ are uncorrelated and have Gaussian distribution then $\mathbf{H}=\mathbf{M}^{-1} \mathbf{K}$ will have Wishart distribution. If $\mathbf{H}$ has a distribution $W_{N}\left(N, \lambda \mathbf{I}_{N}\right)$ then the joint

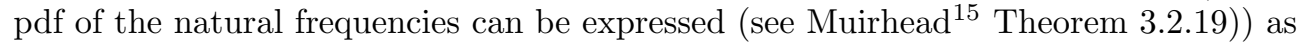

$$
p_{\boldsymbol{\Omega}}\left(\omega_{1}, \omega_{2}, \cdots, \omega_{N}\right)=\frac{\pi^{N^{2} / 2}}{(2 \lambda)^{N^{2} / 2}(\Gamma(N / 2))^{2}} \exp \left(-\frac{1}{2 \lambda} \sum_{i=1}^{N} \omega_{i}^{2}\right) \prod_{i=1}^{N} \frac{1}{\omega_{i}} \prod_{i<j}^{N}\left(\omega_{j}^{2}-\omega_{i}^{2}\right)
$$

Some other exact expressions of the joint probability density function of the eigenvalues of a matrix following more general distributions can be found in the books by Mehta, ${ }^{13}$ Muirhead $^{15}$ and in the paper by Dumitriu and Edelman. ${ }^{21}$ However, unfortunately the system matrices of real structures may not always follow such distributions and consequently some kind of approximate analysis is required. The rest of paper is aimed at developing approximate methods to obtain joint statistics of the natural frequencies.

\section{Statistics of the Natural Frequencies Using Perturbation Methods}

In 1969 using the first-order perturbation method Collins and Thomson ${ }^{14}$ derived the joint probability density function of the eigenvalues and eigenvectors of linear systems with uncertain parameters following Gaussian distribution. Over the past three decades, This work has inspired many authors to apply the firstorder perturbation method in various problems of practical interest, see for example Hasselmen and Hart, ${ }^{22}$ Hart, ${ }^{23}$ Ramu and Ganesan ${ }^{24}$ and Sankar et al. ${ }^{25}$ Adhikari ${ }^{26}$ has used the first-order perturbation method in complex random eigenvalue problems arising in non-proportionally damped systems. The widespread application of the first-order perturbation method is primarily due its computational efficiency compared to other methods. The limitations of this method are well understood - if uncertainties in the system parameters are 'large' so that the 'spread' of the natural frequencies is close to their mean spacings then the method breaks down. This is almost surely the case for higher natural frequencies. Moreover, if the elements of $\mathbf{x}$ are non-Gaussian then the first-order perturbation method is not helpful because there is no general method to obtain the resulting pdf in a simple manner. The accuracy and range of applicability of the first-order perturbation method can be extended if higher-order terms are used. Here we present joint statistics of the natural frequencies using second-order perturbation method. 


\section{A. Perturbation Expansion}

The mass and the stiffness matrices are in general non-linear functions of the random vector $\mathbf{x}$. Denote the mean of $\mathbf{x}$ as $\boldsymbol{\mu} \in \mathbb{R}^{m}$, and consider that

$$
\mathbf{M}(\boldsymbol{\mu})=\overline{\mathbf{M}}, \quad \text { and } \quad \mathbf{K}(\boldsymbol{\mu})=\overline{\mathbf{K}}
$$

are the 'deterministic parts' of the mass and stiffness matrices respectively. In general $\overline{\mathbf{M}}$ and $\overline{\mathbf{K}}$ are different from the mean matrices. The deterministic part of the natural frequencies:

$$
\bar{\omega}_{j}=\omega_{j}(\boldsymbol{\mu})
$$

is obtained from the deterministic eigenvalue problem:

$$
\overline{\mathbf{K}} \bar{\phi}_{j}=\bar{\omega}_{j}^{2} \overline{\mathbf{M}} \bar{\phi}_{j}
$$

The natural frequencies, $\omega_{j}(\mathbf{x}): \mathbb{R}^{m} \mapsto \mathbb{R}$ are non-linear functions of the parameter vector $\mathbf{x}$. If the natural frequencies are not repeated, then each $\omega_{j}(\mathbf{x})$ is expected to be a smooth and twice differentiable function since the mass and stiffness matrices are smooth and twice differentiable functions of the random parameter vector. In the perturbation approach the function $\omega_{j}(\mathbf{x})$ is expanded by its Taylor series about the point $\mathbf{x}=\boldsymbol{\mu}$ as

$$
\omega_{j}(\mathbf{x}) \approx \omega_{j}(\boldsymbol{\mu})+\mathbf{d}_{\omega_{j}}^{T}(\boldsymbol{\mu})(\mathbf{x}-\boldsymbol{\mu})+\frac{1}{2}(\mathbf{x}-\boldsymbol{\mu})^{T} \mathbf{D}_{\omega_{j}}(\boldsymbol{\mu})(\mathbf{x}-\boldsymbol{\mu})
$$

Here $\mathbf{d}_{\omega_{j}}(\boldsymbol{\mu}) \in \mathbb{R}^{m}$ and $\mathbf{D}_{\omega_{j}}(\boldsymbol{\mu}) \in \mathbb{R}^{m \times m}$ are respectively the gradient vector and the Hessian matrix of $\omega_{j}(\mathbf{x})$ evaluated at $\mathbf{x}=\boldsymbol{\mu}$, that is

$$
\begin{aligned}
\left\{\mathbf{d}_{\omega_{j}}(\boldsymbol{\mu})\right\}_{k} & =\left.\frac{\partial \omega_{j}(\mathbf{x})}{\partial x_{k}}\right|_{\mathbf{x}=\boldsymbol{\mu}} \\
\text { and } \quad\left\{\mathbf{D}_{\omega_{j}}(\boldsymbol{\mu})\right\}_{k l} & =\left.\frac{\partial^{2} \omega_{j}(\mathbf{x})}{\partial x_{k} \partial x_{l}}\right|_{\mathbf{x}=\boldsymbol{\mu}}
\end{aligned}
$$

Expressions of the elements of the gradient vector and the Hessian matrix are given in Appendix A. Due to Eq. (16), Eq. (18) implies that the natural frequencies are effectively expanded about their corresponding deterministic values $\bar{\omega}_{j}$.

Equation (18) represents a quadratic form in basic non-Gaussian random variables. The first-order perturbation can be obtained from Eq. (18) by neglecting the Hessian matrix. In this case the natural frequencies are simple linear functions of the basic random variables. This formulation is expected to produce acceptable results when the random variation in $\mathbf{x}$ is small. If the basic random variables are Gaussian then first-order perturbation results in a joint Gaussian distribution of the natural frequencies. ${ }^{14}$ In this case a closed-form expression for their joint probability density function can be obtained easily. When the secondorder terms are retained in Eq. (18) each $\omega_{j}(\mathbf{x})$ results in a quadratic form in $\mathbf{x}$. If the elements of $\mathbf{x}$ are Gaussian then it is possible to obtain joint statistics using theory of quadratic forms as discussed in the next subsection.

\section{B. Joint Statistics of the Natural Frequencies}

Discussions on quadratic forms in Gaussian random variables can be found in the books by Johnson and Kotz $^{27}$ (Chapter 29) and Mathai and Provost. ${ }^{28}$ Using the methods outlined in these references joint moments/cumulants of the natural frequencies are obtained in this section.

Considering $\mathbf{x}$ as multivariate Gaussian random vector with mean $\boldsymbol{\mu} \in \mathbb{R}^{m}$ and covariance matrix $\boldsymbol{\Sigma} \in$ $\mathbb{R}^{m \times m}$, the joint moment generating function of $\omega_{j}(\mathbf{x})$ and $\omega_{k}(\mathbf{x})$, for any $s_{1}, s_{2} \in \mathbb{C}$, can be obtained as

$$
\begin{aligned}
M_{\omega_{j}, \omega_{k}}\left(s_{1}, s_{2}\right) & =\mathrm{E}\left[\exp \left\{s_{1} \omega_{j}(\mathbf{x})+s_{2} \omega_{k}(\mathbf{x})\right\}\right]=\int_{\mathbb{R}^{m}} \exp \left\{s_{1} \omega_{j}(\mathbf{x})+s_{2} \omega_{k}(\mathbf{x})-L(\mathbf{x})\right\} d \mathbf{x} \\
& =(2 \pi)^{-m / 2}\|\boldsymbol{\Sigma}\|^{-1 / 2} \int_{\mathbb{R}^{m}} \exp \left\{s_{1} \omega_{j}(\mathbf{x})+s_{2} \omega_{k}(\mathbf{x})-\frac{1}{2}(\mathbf{x}-\boldsymbol{\mu})^{T} \boldsymbol{\Sigma}^{-1}(\mathbf{x}-\boldsymbol{\mu})\right\} d \mathbf{x}
\end{aligned}
$$


Applying the quadratic expansion in Eq. (18) for $j$ and $k$, and rearranging the terms within the exponent we have

$$
s_{1} \bar{\omega}_{j}+s_{2} \bar{\omega}_{k}+\left(s_{1} \mathbf{d}_{\omega_{j}}+s_{2} \mathbf{d}_{\omega_{k}}\right)^{T}(\mathbf{x}-\boldsymbol{\mu})-\frac{1}{2}(\mathbf{x}-\boldsymbol{\mu})^{T}\left[\boldsymbol{\Sigma}^{-1}-s_{1} \mathbf{D}_{\omega_{j}}-s_{2} \mathbf{D}_{\omega_{k}}\right](\mathbf{x}-\boldsymbol{\mu})
$$

The dependence on $\boldsymbol{\mu}$ has been omitted for notational convenience. Using the transformation $\mathbf{y}=(\mathbf{x}-\boldsymbol{\mu})$ the integral in (21) can be evaluated exactly

$$
\begin{aligned}
& M_{\omega_{j}, \omega_{k}}\left(s_{1}, s_{2}\right)=\left\|\mathbf{I}-s_{1} \mathbf{D}_{\omega_{j}} \boldsymbol{\Sigma}-s_{2} \mathbf{D}_{\omega_{k}} \boldsymbol{\Sigma}\right\|^{-1 / 2} \\
& \quad \exp \left\{\left(s_{1} \bar{\omega}_{j}+s_{2} \bar{\omega}_{k}\right)+\frac{1}{2}\left(s_{1} \mathbf{d}_{\omega_{j}}+s_{2} \mathbf{d}_{\omega_{k}}\right)^{T} \boldsymbol{\Sigma}\left[\mathbf{I}-s_{1} \mathbf{D}_{\omega_{j}} \boldsymbol{\Sigma}-s_{2} \mathbf{D}_{\omega_{k}} \boldsymbol{\Sigma}\right]^{-1}\left(s_{1} \mathbf{d}_{\omega_{j}}+s_{2} \mathbf{d}_{\omega_{k}}\right)\right\}
\end{aligned}
$$

To obtain the joint pdf of $\omega_{j}$ and $\omega_{k}$, the two-dimensional inverse Laplace transform of (23) is required. An exact closed-form expressions for the general case is not possible. Therefore, we calculate the joint cumulants of the natural frequencies.

If first-order perturbation is used then $\mathbf{D}_{\omega_{j}}=\mathbf{D}_{\omega_{k}}=\mathbf{O}$ and from Eq. (23) we obtain

$$
M_{\omega_{j}, \omega_{k}}\left(s_{1}, s_{2}\right)=\exp \left\{\boldsymbol{\mu}_{\omega_{j k}}^{T} \widetilde{\mathbf{s}}+\frac{1}{2} \widetilde{\mathbf{s}}^{T} \boldsymbol{\Sigma}_{\omega_{j k}} \widetilde{\mathbf{s}}\right\}
$$

where

$$
\widetilde{\mathbf{s}}=\left\{\begin{array}{l}
s_{1} \\
s_{2}
\end{array}\right\}, \quad \boldsymbol{\mu}_{\omega_{j k}}=\left\{\begin{array}{c}
\bar{\omega}_{j} \\
\bar{\omega}_{k}
\end{array}\right\}, \quad \text { and } \quad \boldsymbol{\Sigma}_{\omega_{j k}}=\left[\begin{array}{ll}
\mathbf{d}_{\omega_{j}}^{T} \boldsymbol{\Sigma} \mathbf{d}_{\omega_{j}} & \mathbf{d}_{\omega_{j}}^{T} \boldsymbol{\Sigma} \mathbf{d}_{\omega_{k}} \\
\mathbf{d}_{\omega_{j}}^{T} \Sigma \mathbf{d}_{\omega_{k}} & \mathbf{d}_{\omega_{k}}^{T} \boldsymbol{\Sigma} \mathbf{d}_{\omega_{k}}
\end{array}\right]
$$

This implies that $\omega_{j}$ and $\omega_{k}$ are jointly Gaussian distributed with mean $\boldsymbol{\mu}_{\omega_{j k}}$ and covariance matrix $\boldsymbol{\Sigma}_{\omega_{j k}}$.

For the second-order perturbation, the joint cumulants of $\omega_{j}$ and $\omega_{k}$ can be obtained by taking the logarithm of the joint moment generating function (also known as the cumulant generating function)

$$
\begin{aligned}
\ln M_{\omega_{j}, \omega_{k}}\left(s_{1}, s_{2}\right) & =-\frac{1}{2} \ln \left\|\mathbf{I}-s_{1} \mathbf{D}_{\omega_{j}} \boldsymbol{\Sigma}-s_{2} \mathbf{D}_{\omega_{k}} \boldsymbol{\Sigma}\right\| \\
& +\left(s_{1} \bar{\omega}_{j}+s_{2} \bar{\omega}_{k}\right)+\frac{1}{2}\left(s_{1} \mathbf{d}_{\omega_{j}}+s_{2} \mathbf{d}_{\omega_{k}}\right)^{T} \boldsymbol{\Sigma}\left[\mathbf{I}-s_{1} \mathbf{D}_{\omega_{j}} \boldsymbol{\Sigma}-s_{2} \mathbf{D}_{\omega_{k}} \boldsymbol{\Sigma}\right]^{-1}\left(s_{1} \mathbf{d}_{\omega_{j}}+s_{2} \mathbf{d}_{\omega_{k}}\right)
\end{aligned}
$$

A general $\left(r_{1}, r_{2}\right)$-th order cumulant of the $j$ th and $k$ th natural frequencies can be obtained from

$$
\kappa_{j k}^{\left(r_{1}, r_{2}\right)}=\left.\frac{\partial^{r_{1}+r_{2}}}{\partial s_{1}^{r_{1}} \partial s_{2}^{r_{2}}} \ln M_{\omega_{j}, \omega_{k}}\left(s_{1}, s_{2}\right)\right|_{s_{1}=0, s_{2}=0}
$$

Suppose $s_{1}$ and $s_{2}$ are small so that all the eigenvalues of the following matrix

$$
\mathbf{C}=s_{1} \mathbf{D}_{\omega_{j}} \boldsymbol{\Sigma}+s_{2} \mathbf{D}_{\omega_{k}} \boldsymbol{\Sigma}
$$

is less than 1. Then the terms $-\frac{1}{2} \ln \left\|\mathbf{I}-s_{1} \mathbf{D}_{\omega_{j}} \boldsymbol{\Sigma}-s_{2} \mathbf{D}_{\omega_{k}} \boldsymbol{\Sigma}\right\|$ and $\left[\mathbf{I}-s_{1} \mathbf{D}_{\omega_{j}} \boldsymbol{\Sigma}-s_{2} \mathbf{D}_{\omega_{k}} \boldsymbol{\Sigma}\right]^{-1}$ appearing in Eq. (26) can be expanded in a Taylor series as

$$
\begin{array}{ll} 
& -\frac{1}{2} \ln \|\mathbf{I}-\mathbf{C}\|=\operatorname{Trace}(\mathbf{C})+\frac{1}{2} \operatorname{Trace}\left(\mathbf{C}^{2}\right)+\frac{1}{3} \operatorname{Trace}\left(\mathbf{C}^{3}\right)+\cdots \\
\text { and } \quad & {[\mathbf{I}-\mathbf{C}]^{-1}=\mathbf{I}+\mathbf{C}+\mathbf{C}^{2}+\cdots}
\end{array}
$$

Using these expansions, the cumulant generating function in (26) can be expressed by a polynomial in $s_{1}$ and $s_{2}$ and therefore the cumulants can be obtained from Eq. (27) in a straightforward manner. After some simplifications the following results can be derived

$$
\begin{aligned}
\kappa_{j k}^{(1,0)} & =\mathrm{E}\left[\omega_{j}\right]=\bar{\omega}_{j}+\frac{1}{2} \operatorname{Trace}\left(\mathbf{D}_{\omega_{j}} \boldsymbol{\Sigma}\right), \\
\kappa_{j k}^{(0,1)} & =\mathrm{E}\left[\omega_{k}\right]=\bar{\omega}_{k}+\frac{1}{2} \operatorname{Trace}\left(\mathbf{D}_{\omega_{k}} \boldsymbol{\Sigma}\right), \\
\kappa_{j k}^{(1,1)} & =\operatorname{Cov}\left(\omega_{j}, \omega_{k}\right)=\frac{1}{2} \operatorname{Trace}\left(\left(\mathbf{D}_{\omega_{j}} \boldsymbol{\Sigma}\right)\left(\mathbf{D}_{\omega_{k}} \boldsymbol{\Sigma}\right)\right)+\mathbf{d}_{\omega_{j}}^{T} \boldsymbol{\Sigma} \mathbf{d}_{\omega_{k}}
\end{aligned}
$$


The general case can be obtained following Mathai and Provost ${ }^{28}$ (see Chapter 3) as

$$
\begin{aligned}
& \kappa_{j k}^{\left(r_{1}, r_{2}\right)}=\frac{1}{2}\left(r_{1}+r_{2}-2\right) !\left\{\left(r_{1}\right.\right.\left.+r_{2}-1\right) \operatorname{Trace}\left(\left(\mathbf{D}_{\omega_{j}} \boldsymbol{\Sigma}\right)^{r_{1}}\left(\mathbf{D}_{\omega_{k}} \boldsymbol{\Sigma}\right)^{r_{2}}\right) \\
&+r_{1}\left(r_{1}-1\right) \mathbf{d}_{\omega_{j}}^{T}\left(\mathbf{D}_{\omega_{j}} \boldsymbol{\Sigma}\right)^{r_{1}-2}\left(\mathbf{D}_{\omega_{k}} \boldsymbol{\Sigma}\right)^{r_{2}} \boldsymbol{\Sigma} \mathbf{d}_{\omega_{j}}+r_{2}\left(r_{2}-1\right) \mathbf{d}_{\omega_{k}}^{T}\left(\mathbf{D}_{\omega_{j}} \boldsymbol{\Sigma}\right)^{r_{1}}\left(\mathbf{D}_{\omega_{k}} \boldsymbol{\Sigma}\right)^{r_{2}-2} \boldsymbol{\Sigma} \mathbf{d}_{\omega_{k}} \\
&\left.+2 r_{1} r_{2} \mathbf{d}_{\omega_{j}}^{T}\left(\mathbf{D}_{\omega_{j}} \boldsymbol{\Sigma}\right)^{r_{1}-1}\left(\mathbf{D}_{\omega_{k}} \boldsymbol{\Sigma}\right)^{r_{2}-1} \boldsymbol{\Sigma} \mathbf{d}_{\omega_{k}}\right\}, \quad \text { for } \quad r_{1} \geq 1, r_{2} \geq 1
\end{aligned}
$$

Since all the cumulants are known from the preceding expressions, the joint pdf of the natural frequencies can be calculated, for example using Edgeworth expansion, up to any desired accuracy. Recall that the limitation of this approach arises from the second-order Taylor expansion of the natural frequencies around the deterministic values. Therefore, the inclusion of higher order cumulants in the expression of the joint pdf will not overcome this fundamental limitation.

The method described in this section is only applicable when the basic random variables are Gaussian. When the elements of $\mathbf{x}$ are non-Gaussian then neither the first-order perturbation nor the second-order perturbation methods are helpful because there is no general method to obtain the resulting statistics in a simple manner. In such cases the method outlined in the next section might be useful.

\section{Statistics of the Natural Frequencies Using Asymptotic Integral Based Method}

\section{A. Multidimensional Integrals in Unbounded Domains}

In this section the moments of the natural frequencies are obtained based on an asymptotic approximation of the multidimensional integral. Consider a function $f(\mathbf{x}): \mathbb{R}^{m} \mapsto \mathbb{R}$ which is smooth and at least twice differentiable. Suppose we want to evaluate an integral of the following form:

$$
\mathcal{J}=\int_{\mathbb{R}^{m}} \exp \{-f(\mathbf{x})\} d \mathbf{x}
$$

This is an $m$-dimensional integral over the unbounded domain $\mathbb{R}^{m}$. The maximum contribution to this integral comes from the neighborhood where $f(\mathbf{x})$ reaches its global minimum. Suppose that $f(\mathbf{x})$ reaches its global minimum at an unique point $\boldsymbol{\theta} \in \mathbb{R}^{m}$. Therefore, at $\mathbf{x}=\boldsymbol{\theta}$

$$
\frac{\partial f(\mathbf{x})}{\partial x_{k}}=0, \forall k \quad \text { or } \quad \mathbf{d}_{f}(\boldsymbol{\theta})=\mathbf{0}
$$

Using this, expand $f(\mathbf{x})$ in a Taylor series about $\boldsymbol{\theta}$ and rewrite Eq. (35) as

$$
\begin{aligned}
\mathcal{J} & =\int_{\mathbb{R}^{m}} \exp \left\{-\left\{f(\boldsymbol{\theta})+\frac{1}{2}(\mathbf{x}-\boldsymbol{\theta})^{T} \mathbf{D}_{f}(\boldsymbol{\theta})(\mathbf{x}-\boldsymbol{\theta})+\varepsilon(\mathbf{x}, \boldsymbol{\theta})\right\}\right\} d \mathbf{x} \\
& =\exp \{-f(\boldsymbol{\theta})\} \int_{\mathbb{R}^{m}} \exp \left\{-\frac{1}{2}(\mathbf{x}-\boldsymbol{\theta})^{T} \mathbf{D}_{f}(\boldsymbol{\theta})(\mathbf{x}-\boldsymbol{\theta})-\varepsilon(\mathbf{x}, \boldsymbol{\theta})\right\} d \mathbf{x}
\end{aligned}
$$

where $\varepsilon(\mathbf{x}, \boldsymbol{\theta})$ is the error if only the terms up to second-order were retained in the Taylor series expansion. With suitable scaling of $\mathbf{x}$ the integral in (35) can be transformed to the so called 'Laplace integral'. Under special conditions such integrals can be well approximated using asymptotic methods. The relevant mathematical methods and formal derivations are covered in detail in the books by Bleistein and Handelsman ${ }^{29}$ and Wong. ${ }^{30}$ Here we propose a somewhat different version of asymptotic integrals. The error $\varepsilon(\mathbf{x}, \boldsymbol{\theta})$ depends on higher order derivatives of $f(\mathbf{x})$ at $\mathbf{x}=\boldsymbol{\theta}$. If they are small compared to $f(\boldsymbol{\theta})$ and the elements of $\mathbf{D}_{f}(\boldsymbol{\theta})$, their contribution will be negligible to the value of the integral. Therefore, we assume $f(\boldsymbol{\theta})$ and the elements of $\mathbf{D}_{f}(\boldsymbol{\theta})$ are large so that

$$
\left|\frac{1}{f(\boldsymbol{\theta})} \mathcal{D}^{(j)}(f(\boldsymbol{\theta}))\right| \rightarrow 0 \quad \text { and } \quad \forall k, l,\left|\frac{1}{\left[\mathbf{D}_{f}(\boldsymbol{\theta})\right]_{k l}} \mathcal{D}^{(j)}(f(\boldsymbol{\theta}))\right| \rightarrow 0 \quad \text { for } \quad j>2
$$

where $\mathcal{D}^{(j)}(f(\boldsymbol{\theta}))$ is $j$ th order derivative of $f(\mathbf{x})$ evaluated at $\mathbf{x}=\boldsymbol{\theta}$. Under such assumptions $\varepsilon(\mathbf{x}, \boldsymbol{\theta}) \rightarrow 0$. Therefore, the integral in $(37)$ can be approximated as

$$
\begin{gathered}
\mathcal{J} \approx \exp \{-f(\boldsymbol{\theta})\} \int_{\mathbb{R}^{m}} \exp \left\{-\frac{1}{2}(\mathbf{x}-\boldsymbol{\theta})^{T} \mathbf{D}_{f}(\boldsymbol{\theta})(\mathbf{x}-\boldsymbol{\theta})\right\} d \mathbf{x} \\
7 \text { of } 18
\end{gathered}
$$


If $\boldsymbol{\theta}$ is the global minima of $f(\mathbf{x})$ in $\mathbb{R}^{m}$, the symmetric Hessian matrix $\mathbf{D}_{f}(\boldsymbol{\theta}) \in \mathbb{R}^{m \times m}$ is also expected to be positive definite. Now use the coordinate transformation

$$
\boldsymbol{\xi}=(\mathbf{x}-\boldsymbol{\theta}) \mathbf{D}_{f}^{-1 / 2}(\boldsymbol{\theta})
$$

The Jacobian of this transformation is

$$
\|\mathbf{J}\|=\left\|\mathbf{D}_{f}(\boldsymbol{\theta})\right\|^{-1 / 2}
$$

Using Eq. (40), the integral in Eq. (39) can be evaluated as

$$
\begin{aligned}
\mathcal{J} & \approx \exp \{-f(\boldsymbol{\theta})\} \int_{\mathbb{R}^{m}}\left\|\mathbf{D}_{f}(\boldsymbol{\theta})\right\|^{-1 / 2} \exp \left\{-\frac{1}{2}\left(\boldsymbol{\xi}^{T} \boldsymbol{\xi}\right)\right\} d \boldsymbol{\xi} \\
\text { or } \mathcal{J} & \approx(2 \pi)^{m / 2} \exp \{-f(\boldsymbol{\theta})\}\left\|\mathbf{D}_{f}(\boldsymbol{\theta})\right\|^{-1 / 2}
\end{aligned}
$$

A similar approach was outlined by Papadimitriou et. al. ${ }^{31}$ for the calculation of response moments of a dynamic system. This analysis proposed here is somewhat different from the widely used Laplace's method of asymptotic approximation of integrals (Wong, ${ }^{30}$ Chapter IX, Theorem 3). Here it is simply assumed that the higher order derivatives $\mathcal{D}^{(j)}(f(\boldsymbol{\theta}))$ are negligibly small compared to $f(\boldsymbol{\theta})$ and the elements of $\mathbf{D}_{f}(\boldsymbol{\theta})$. This approximation is expected to yield good result if the minima of $f(\mathbf{x})$ around $\mathbf{x}=\boldsymbol{\theta}$ is sharp. If $\boldsymbol{\theta}$ is not unique then it is required to sum the contributions arising from all such optimal points separately. Equation (43) will now be used to obtain joint moments of the natural frequencies.

\section{B. Joint Moments of Two Natural Frequencies}

A general $\left(r_{1}, r_{2}\right)$-th order joint moment of two natural frequencies $\omega_{j}$ and $\omega_{k}$ can be expressed as

$$
\begin{aligned}
\mu_{j k}^{\left(r_{1}, r_{2}\right)} & =\mathrm{E}\left[\omega_{j}^{r_{1}}(\mathbf{x}) \omega_{k}^{r_{2}}(\mathbf{x})\right]=\int_{\mathbb{R}^{m}} \omega_{j}^{r_{1}}(\mathbf{x}) \omega_{k}^{r_{2}}(\mathbf{x}) p_{\mathbf{x}}(\mathbf{x}) d \mathbf{x} \\
& =\int_{\mathbb{R}^{m}} \exp \left\{-\left(L(\mathbf{x})-r_{1} \ln \omega_{j}(\mathbf{x})-r_{2} \ln \omega_{k}(\mathbf{x})\right)\right\} d \mathbf{x}, \quad r_{1}, r_{2}=1,2,3 \cdots
\end{aligned}
$$

The equation can be expressed in the form of Eq. (35) by choosing

$$
f(\mathbf{x})=L(\mathbf{x})-r_{1} \ln \omega_{j}(\mathbf{x})-r_{2} \ln \omega_{k}(\mathbf{x})
$$

Differentiating the above equation with respect to $x_{i}$ we obtain

$$
\frac{\partial f(\mathbf{x})}{\partial x_{i}}=\frac{\partial L(\mathbf{x})}{\partial x_{i}}-\frac{r_{1}}{\omega_{j}(\mathbf{x})} \frac{\partial \omega_{j}(\mathbf{x})}{\partial x_{i}}-\frac{r_{2}}{\omega_{k}(\mathbf{x})} \frac{\partial \omega_{k}(\mathbf{x})}{\partial x_{i}}
$$

The optimal point $\boldsymbol{\theta}$ can be obtained from $(\overline{36})$ by equating the above expression to zero. Therefore at $\mathbf{x}=\boldsymbol{\theta}$

$$
\begin{gathered}
\frac{\partial f(\mathbf{x})}{\partial x_{i}}=0, \quad \forall i \\
\text { or } \frac{r_{1}}{\omega_{j}(\mathbf{x})} \frac{\partial \omega_{j}(\mathbf{x})}{\partial x_{i}}+\frac{r_{2}}{\omega_{k}(\mathbf{x})} \frac{\partial \omega_{k}(\mathbf{x})}{\partial x_{i}}=\frac{\partial L(\boldsymbol{\theta})}{\partial x_{i}}, \quad \forall k \\
\text { or } \quad \mathbf{d}_{L}(\boldsymbol{\theta})=\frac{r_{1}}{\omega_{j}(\boldsymbol{\theta})} \mathbf{d}_{\omega_{j}}(\boldsymbol{\theta})+\frac{r_{2}}{\omega_{k}(\boldsymbol{\theta})} \mathbf{d}_{\omega_{k}}(\boldsymbol{\theta})
\end{gathered}
$$

The elements of the Hessian matrix $\mathbf{D}_{f}(\boldsymbol{\theta})$ can be obtained by differentiating Eq. (46) with respect to $x_{l}$ :

$$
\frac{\partial^{2} f(\mathbf{x})}{\partial x_{i} \partial x_{l}}=\frac{\partial^{2} L(\mathbf{x})}{\partial x_{i} \partial x_{l}}+\frac{r_{1}}{\omega_{j}^{2}(\mathbf{x})} \frac{\partial \omega_{j}(\mathbf{x})}{\partial x_{l}} \frac{\partial \omega_{j}(\mathbf{x})}{\partial x_{i}}-\frac{r_{1}}{\omega_{j}(\mathbf{x})} \frac{\partial^{2} \omega_{j}(\mathbf{x})}{\partial x_{i} \partial x_{l}}+\frac{r_{2}}{\omega_{k}^{2}(\mathbf{x})} \frac{\partial \omega_{k}(\mathbf{x})}{\partial x_{l}} \frac{\partial \omega_{k}(\mathbf{x})}{\partial x_{i}}-\frac{r_{2}}{\omega_{k}(\mathbf{x})} \frac{\partial^{2} \omega_{k}(\mathbf{x})}{\partial x_{i} \partial x_{l}}
$$

Combining this equation for all $i$ and $l$ we have

$$
\mathbf{D}_{f}(\boldsymbol{\theta})=\mathbf{D}_{L}(\boldsymbol{\theta})+\frac{r_{1}}{\omega_{j}^{2}(\boldsymbol{\theta})} \mathbf{d}_{\omega_{j}}(\boldsymbol{\theta}) \mathbf{d}_{\omega_{j}}^{T}(\boldsymbol{\theta})-\frac{r_{1}}{\omega_{j}(\boldsymbol{\theta})} \mathbf{D}_{\omega_{j}}(\boldsymbol{\theta})+\frac{r_{2}}{\omega_{k}^{2}(\boldsymbol{\theta})} \mathbf{d}_{\omega_{k}}(\boldsymbol{\theta}) \mathbf{d}_{\omega_{k}}^{T}(\boldsymbol{\theta})-\frac{r_{2}}{\omega_{k}(\boldsymbol{\theta})} \mathbf{D}_{\omega_{k}}(\boldsymbol{\theta})
$$


Using the asymptotic approximation (43), the joint moment of two natural frequencies can be obtained as

$$
\mu_{j k}^{\left(r_{1}, r_{2}\right)} \approx(2 \pi)^{m / 2} \omega_{j}^{r_{1}}(\boldsymbol{\theta}) \omega_{k}^{r_{2}}(\boldsymbol{\theta}) \exp \{-L(\boldsymbol{\theta})\}\left\|\mathbf{D}_{f}(\boldsymbol{\theta})\right\|^{-1 / 2}
$$

The optimal point $\boldsymbol{\theta}$ needs to be calculated by solving non-linear set of equations Eq. (49). Since explicit analytical expression of $\mathbf{d}_{\omega_{j}}$ in terms of the derivative of the mass and stiffness matrices is avaliable, ${ }^{32}$ expensive numerical differentiation of $\omega_{j}(\mathbf{x})$ at each step is not needed. Moreover, for most $p_{\mathbf{x}}(\mathbf{x})$, a closedform expression of $\mathbf{d}_{L}(\mathbf{x})$ is available. If $\mathbf{d}_{L}(\mathbf{x})$ is given by (3), then a simple iterative method can be used ${ }^{11,12}$ to obtain $\boldsymbol{\theta}$. The mean of the natural frequencies can be obtained by substituting $r_{1}=0$ or $r_{2}=0$ in Eq. $(52)$ as

$$
\mathrm{E}\left[\omega_{j}\right]=\mu_{j k}^{(1,0)} \approx(2 \pi)^{m / 2} \omega_{j}(\boldsymbol{\theta}) \exp \{-L(\boldsymbol{\theta})\}\left\|\mathbf{D}_{f}(\boldsymbol{\theta})\right\|^{-1 / 2}
$$

where $\boldsymbol{\theta}$ is obtained from

$$
\begin{aligned}
\quad \mathbf{d}_{L}(\boldsymbol{\theta}) & =\mathbf{d}_{\omega_{j}}(\boldsymbol{\theta}) / \omega_{j}(\boldsymbol{\theta}) \\
\text { and } \quad \mathbf{D}_{f}(\boldsymbol{\theta}) & =\mathbf{D}_{L}(\boldsymbol{\theta})+\left(\mathbf{d}_{\omega_{j}}(\boldsymbol{\theta}) \mathbf{d}_{\omega_{j}}^{T}(\boldsymbol{\theta}) / \omega_{j}(\boldsymbol{\theta})-\mathbf{D}_{\omega_{j}}(\boldsymbol{\theta})\right) / \omega_{j}(\boldsymbol{\theta})
\end{aligned}
$$

The elements of the covariance matrix of the natural frequencies can obtained as

$$
\operatorname{Cov}\left(\omega_{j}, \omega_{k}\right)=\mathrm{E}\left[\left(\omega_{j}-\mathrm{E}\left[\omega_{j}\right]\right)\left(\omega_{k}-\mathrm{E}\left[\omega_{k}\right]\right)\right]=\mu_{j k}^{(1,1)}-\mu_{j k}^{(1,0)} \mu_{j k}^{(0,1)}
$$

\section{Arbitrary Joint Moments of Multiple Natural Frequencies}

The formulation presented in the previous subsection can be readily generalized to obtain arbitrary order joint moments of multiple natural frequencies. We want to obtain

$$
\mu_{j_{1} j_{2} \cdots j_{n}}^{\left(r_{1}, r_{2}, \cdots r_{n}\right)}=\int_{\mathbb{R}^{m}}\left\{\omega_{j_{1}}^{r_{1}}(\mathbf{x}) \omega_{j_{2}}^{r_{2}}(\mathbf{x}) \cdots \omega_{j_{n}}^{r_{n}}(\mathbf{x})\right\} p_{\mathbf{x}}(\mathbf{x}) d \mathbf{x}
$$

By choosing the function

$$
f(\mathbf{x})=L(\mathbf{x})-r_{1} \ln \omega_{j_{1}}(\mathbf{x})-r_{2} \ln \omega_{j_{2}}(\mathbf{x})-\cdots r_{n} \ln \omega_{j_{n}}(\mathbf{x})
$$

and applying the asymptotic approximation in Eq. (43) it can be shown that

$$
\mu_{j_{1} j_{2} \cdots j_{n}}^{\left(r_{1}, r_{2}, \cdots r_{n}\right)} \approx(2 \pi)^{m / 2}\left\{\omega_{j_{1}}^{r_{1}}(\boldsymbol{\theta}) \omega_{j_{2}}^{r_{2}}(\boldsymbol{\theta}) \cdots \omega_{j_{n}}^{r_{n}}(\boldsymbol{\theta})\right\} \exp \{-L(\boldsymbol{\theta})\}\left\|\mathbf{D}_{f}(\boldsymbol{\theta})\right\|^{-1 / 2}
$$

where $\boldsymbol{\theta}$ is obtained by solving

$$
\mathbf{d}_{L}(\boldsymbol{\theta})=\frac{r_{1}}{\omega_{j_{1}}(\boldsymbol{\theta})} \mathbf{d}_{\omega_{j_{1}}}(\boldsymbol{\theta})+\frac{r_{2}}{\omega_{j_{2}}(\boldsymbol{\theta})} \mathbf{d}_{\omega_{j_{2}}}(\boldsymbol{\theta})+\cdots \frac{r_{n}}{\omega_{j_{n}}(\boldsymbol{\theta})} \mathbf{d}_{\omega_{j_{n}}}(\boldsymbol{\theta})
$$

and the Hessian matrix is given by

$$
\mathbf{D}_{f}(\boldsymbol{\theta})=\mathbf{D}_{L}(\boldsymbol{\theta})+\sum_{\substack{j=j_{1}, j_{2}, \cdots \\ r=r_{1}, r_{2}, \cdots}}^{j_{n}, r_{n}} \frac{r}{\omega_{j}^{2}(\boldsymbol{\theta})} \mathbf{d}_{\omega_{j}}(\boldsymbol{\theta}) \mathbf{d}_{\omega_{j}}^{T}(\boldsymbol{\theta})-\frac{r}{\omega_{j}(\boldsymbol{\theta})} \mathbf{D}_{\omega_{j}}(\boldsymbol{\theta})
$$

Eq. (59) is perhaps the most general formula to obtain the moments of the natural frequencies of linear stochastic dynamic systems. Once the joint moments are known, the joint probability density functions of the natural frequencies are obtained, for example, using the maximum entropy principle. Proposed method is applied to dynamic analysis of a 3 dof system with uncertain properties. 


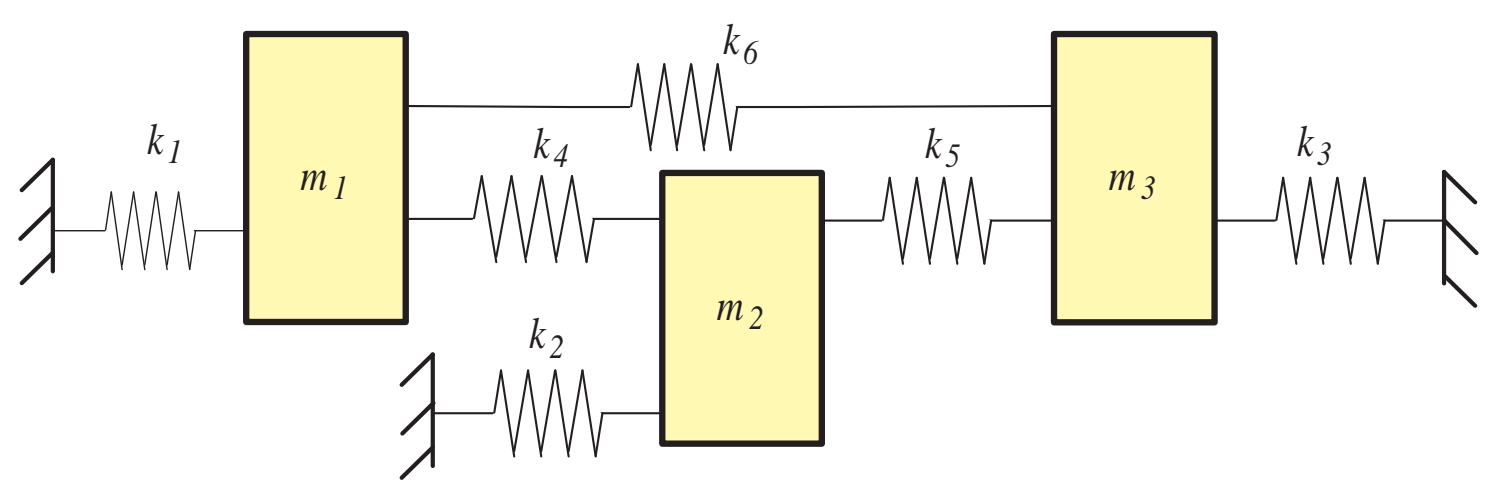

Figure 1. The three degree-of-freedom random system.

\section{Numerical Examples}

\section{A. System model and computational methodology}

A three-degree-of-freedom undamped spring-mass system, taken from reference, ${ }^{33}$ is shown in figure 1 . The mass and stiffness matrices of the example system are given by

$$
\mathbf{M}=\left[\begin{array}{ccc}
m_{1} & 0 & 0 \\
0 & m_{2} & 0 \\
0 & 0 & m_{3}
\end{array}\right] \quad \text { and } \quad \mathbf{K}=\left[\begin{array}{ccc}
k_{1}+k_{4}+k_{6} & -k_{4} & -k_{6} \\
-k_{4} & k_{4}+k_{5}+k_{6} & -k_{5} \\
-k_{6} & -k_{5} & k_{5}+k_{3}+k_{6}
\end{array}\right]
$$

It is assumed that all mass and stiffness constants are random. The randomness in these parameters are assumed to be of the following form:

$$
\begin{array}{ll}
m_{i}=\bar{m}_{i}\left(1+\epsilon_{m} x_{i}\right), & i=1,2,3 \\
k_{i}=\bar{k}_{i}\left(1+\epsilon_{k} x_{i+3}\right), & i=1, \cdots, 6
\end{array}
$$

Here $\mathbf{x}=\left\{x_{1}, \cdots, x_{9}\right\}^{T} \in \mathbb{R}^{9}$ is the vector of random variables. It is assumed that all random variables are Gaussian and uncorrelated with zero mean and unit standard deviation, that is $\boldsymbol{\mu}=\mathbf{0}$ and $\boldsymbol{\Sigma}=\mathbf{I}$. Therefore, the mean values of $m_{i}$ and $k_{i}$ are given by $\bar{m}_{i}$ and $\bar{k}_{i}$. The numerical values of the constants are assumed to be $\bar{m}_{i}=1.0 \mathrm{~kg}$ for $i=1,2,3 ; \bar{k}_{i}=1.0 \mathrm{~N} / \mathrm{m}$ for $i=1, \cdots, 5$ and $k_{6}=3.0 \mathrm{~N} / \mathrm{m}$. The numerical values of the 'strength parameters' are assumed to be $\epsilon_{m}=0.15$ and $\epsilon_{k}=0.20$. In order to obtain the statistics of the natural frequencies using the methods developed in this paper, the gradient vector and the Hessian matrix of the natural frequencies are required. As shown in Appendix $\mathrm{A}$, this in turn requires the derivative of the system matrices with respect to the entries of $\mathbf{x}$. For most practical problems, which usually involve Finite Element modeling, these derivatives need to be determined numerically. However, for this simple example the derivatives can be obtained in closed-form and they are given in Appendix $\mathrm{B}$.

We calculate joint moments and joint probability density functions of the natural frequencies of the system. Attention is restricted up to second-order joint statistics of two natural frequencies. Following four methods are used to obtain the joint moments and the joint probability density functions:

1. First-order perturbation: For this case the mean and covariance matrix of the natural frequencies are calculated using Eqs. (31) and (33) by substituting the Hessian matrices $\mathbf{D}_{\omega_{j}}=\mathbf{O}$ and $\mathbf{D}_{\omega_{k}}=\mathbf{O}$. Recalling that for this problem $\boldsymbol{\Sigma}=\mathbf{I}$, the resulting statistics for this special case can be obtained as

$$
\begin{aligned}
\mathrm{E}[\omega]_{j} & =\bar{\omega}_{j} \\
\text { and } \quad \operatorname{Cov}\left(\omega_{j}, \omega_{k}\right) & =\mathbf{d}_{\omega_{j}}^{T} \mathbf{d}_{\omega_{k}}
\end{aligned}
$$

The gradient vector $\mathbf{d}_{\omega_{j}}$ can be obtained from Eq. (77) using the system derivative matrices (81) and (82). 
2. Second-order perturbation: In this case the Hessian matrices $\mathbf{D}_{\omega_{j}}$ and $\mathbf{D}_{\omega_{k}}$ are used in calculating the joint statistics of the natural frequencies using Eqs. (31) and (33). The elements of the Hessian matrices $\mathbf{D}_{\omega_{j}}$ and $\mathbf{D}_{\omega_{k}}$ can be calculated using Eq. (79). The resulting statistics for this special case can be obtained as

$$
\begin{aligned}
\mathrm{E}[\omega]_{j} & =\bar{\omega}_{j}+\frac{1}{2} \operatorname{Trace}\left(\mathbf{D}_{\omega_{j}}\right) \\
\text { and } \operatorname{Cov}\left(\omega_{j}, \omega_{k}\right) & =\mathbf{d}_{\omega_{j}}^{T} \mathbf{d}_{\omega_{k}}+\frac{1}{2} \operatorname{Trace}\left(\mathbf{D}_{\omega_{j}} \mathbf{D}_{\omega_{k}}\right)
\end{aligned}
$$

Comparing these results with Eqs. (65) and (66), the contributions of the Hessian matrices can be regarded as corrections to the first-order perturbation results.

3. Method based on the asymptotic integral: In this case the mean and covariance matrix of the natural frequencies are calculated using Eqs. (53) and (56). The function $L(\mathbf{x})$ can be obtained by substituting $\boldsymbol{\mu}=\mathbf{0}$ and $\boldsymbol{\Sigma}=\mathbf{I}$ in Eq. (3) as

$$
L(\mathbf{x})=\frac{m}{2} \ln (2 \pi)+\frac{1}{2} \mathbf{x}^{T} \mathbf{x}
$$

The gradient vector and the Hessian matrix of $L(\mathbf{x})$, needed to apply this method, are given by

$$
\mathbf{d}_{L}(\mathbf{x})=\mathbf{x} \quad \text { and } \quad \mathbf{D}_{L}(\mathbf{x})=\mathbf{I}
$$

4. Monte Carlo Simulation (MCS): The samples of the nine independent Gaussian random variables $x_{i}, i=1, \cdots, 9$ are generated and the natural frequencies are computed directly from Eq. (1). A total of 15000 samples are used to obtain the statistical moments and histograms of the pdf of the natural frequencies. The results obtained from MCS are assumed to be the benchmark for the purpose of comparing the analytical methods.

The results are presented and discussed in the next subsection.

\section{B. Numerical results}

For the given parameter values the natural frequencies (in $\mathrm{rad} / \mathrm{s}$ ) of the corresponding deterministic system is given by

$$
\bar{\omega}_{1}=1, \quad \bar{\omega}_{2}=2, \quad \text { and } \quad \bar{\omega}_{3}=3
$$

Figure 2 shows percentage error with respect to Monte Carlo Simulation in the elements of the mean vector and covariance matrix of the natural frequencies. Since the covariance matrix is a symmetric matrix, only

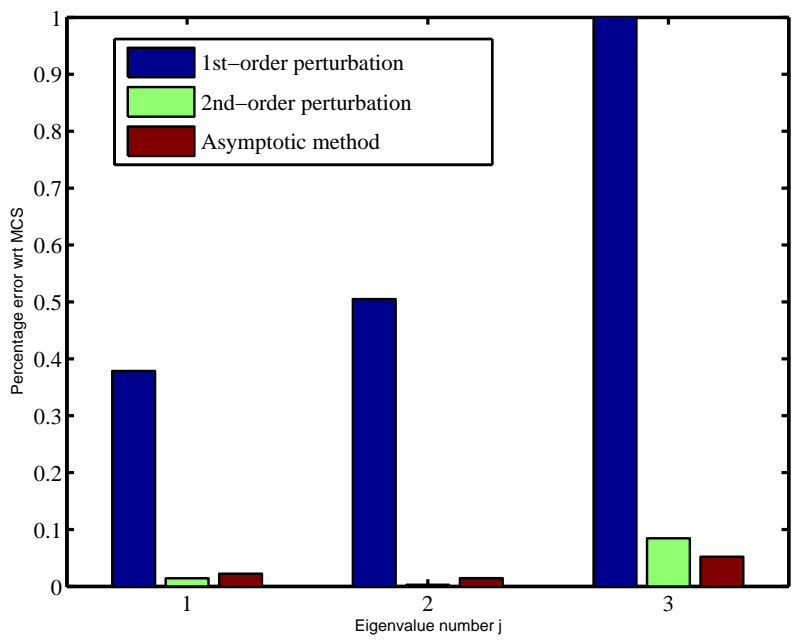

(a) Error in the mean values.

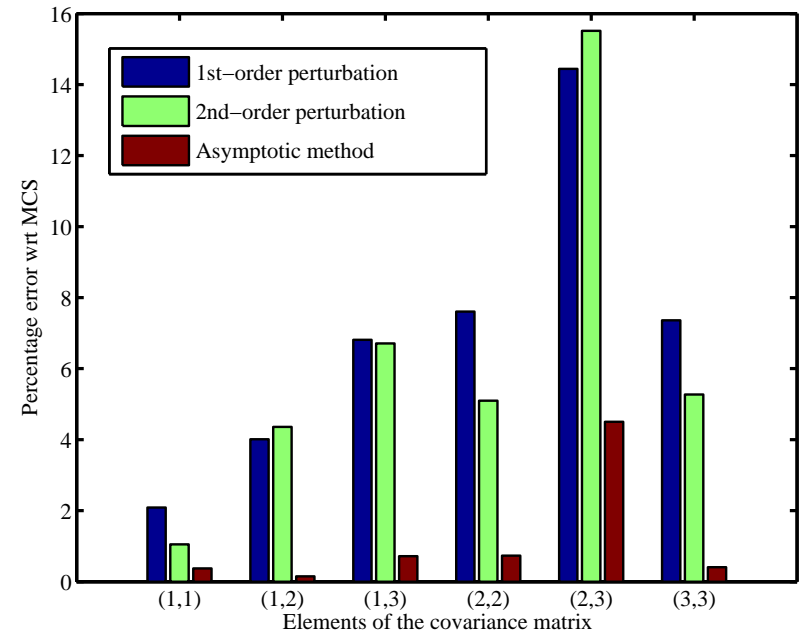

(b) Error in the elements of the covariance matrix.

Figure 2. Percentage error with respect to MCS in the mean and covariance matrix of the natural frequencies. 
the elements of the upper triangular part is considered for plotting. For the mean values, the first-order perturbation method is the least accurate, followed by the second-order perturbation method. The same fact is also true for the diagonal elements of the covariance matrix (that is the variance of the natural frequencies). However, for the off-diagonal terms, the second-order perturbation method appears to be slightly less accurate compared to the first-order perturbation method. For both calculations, the asymptotic method is clearly the most accurate among the three analytical methods used in this study.

Now consider the probability density function of the natural frequencies. Because the asymptotic method is the most accurate among the three methods discussed here, we will only persue this method in the remaining discussions. First we focus on the marginal pdf of the natural frequencies. Using the asymptotic method, the mean and standard deviation of the natural frequencies are obtained as

$$
\begin{aligned}
\boldsymbol{\mu}_{\boldsymbol{\Omega}} & =\{0.9962,2.0102,3.0312\}^{T} \\
\text { and } \quad \boldsymbol{\sigma}_{\boldsymbol{\Omega}} & =\{0.0729,0.1603,0.2955\}^{T}
\end{aligned}
$$

Gaussian distributions are fitted with these parameters and compared with Monte Carlo Simulation. The marginal pdf of the natural frequencies obtained from the asymptotic method and Monte Carlo Simulation are shown in figure 3 . Each MCS pdf in figure 3 is obtained by normalizing the histogram of the samples so that the area under the curve obtained by joining the middle points of the histogram bins is equal to unity. The Gaussian distributions calculated from the asymptotic method fit quite well to the Monte Carlo Simulation. This result implies that the probability density function of the individual natural frequencies can be approximated well using a Gaussian distribution with correct set of parameters.

Now we focus on the joint distribution

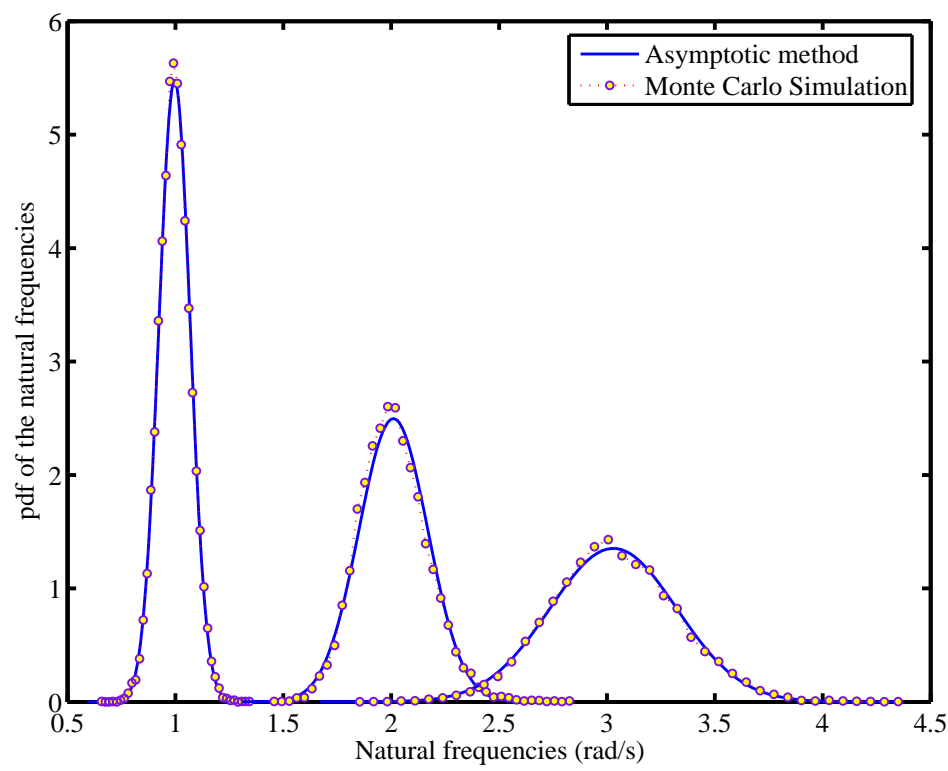

Figure 3. Probability density function of the natural frequencies.

of $\omega_{1}$ being further from $\omega_{3}$ compared to $\omega_{2}$.

In line with the univariate Gaussian distributions shown in figure 3, we can obtain bivariate Gaussian distribution for each pair of natural frequencies. Joint probability density function of the natural frequencies obtained from the asymptotic method and Monte Carlo Simulation are shown in figures 4 and [5. In total three joint distributions, namely $p_{\omega_{1}, \omega_{2}}, p_{\omega_{1}, \omega_{3}}$ and $p_{\omega_{2}, \omega_{3}}$ are shown in figures 4 and 5 . Each analytical joint pdf in figure 4 is obtained by fitting a bivariate Gaussian distribution with the mean vector and covariance matrix taken from Eqs. (72) and (74) for the corresponding set of natural frequencies. The MCS pdf in figure 5 is obtained by normalizing the two dimensional histogram of the samples so that the volume under the surface obtained by joining the middle points of the histogram bins is equal to unity. At first it may appear that, like the marginal pdfs in figure 3, the joint pdfs of the natural frequencies are also jointly Gaussian distributed. But a closer inspection reveals that this is not always the case. Figure 6 compares the contours of the analytical joint pdf with that obtained from MCS. The adjacent natural frequencies, that 


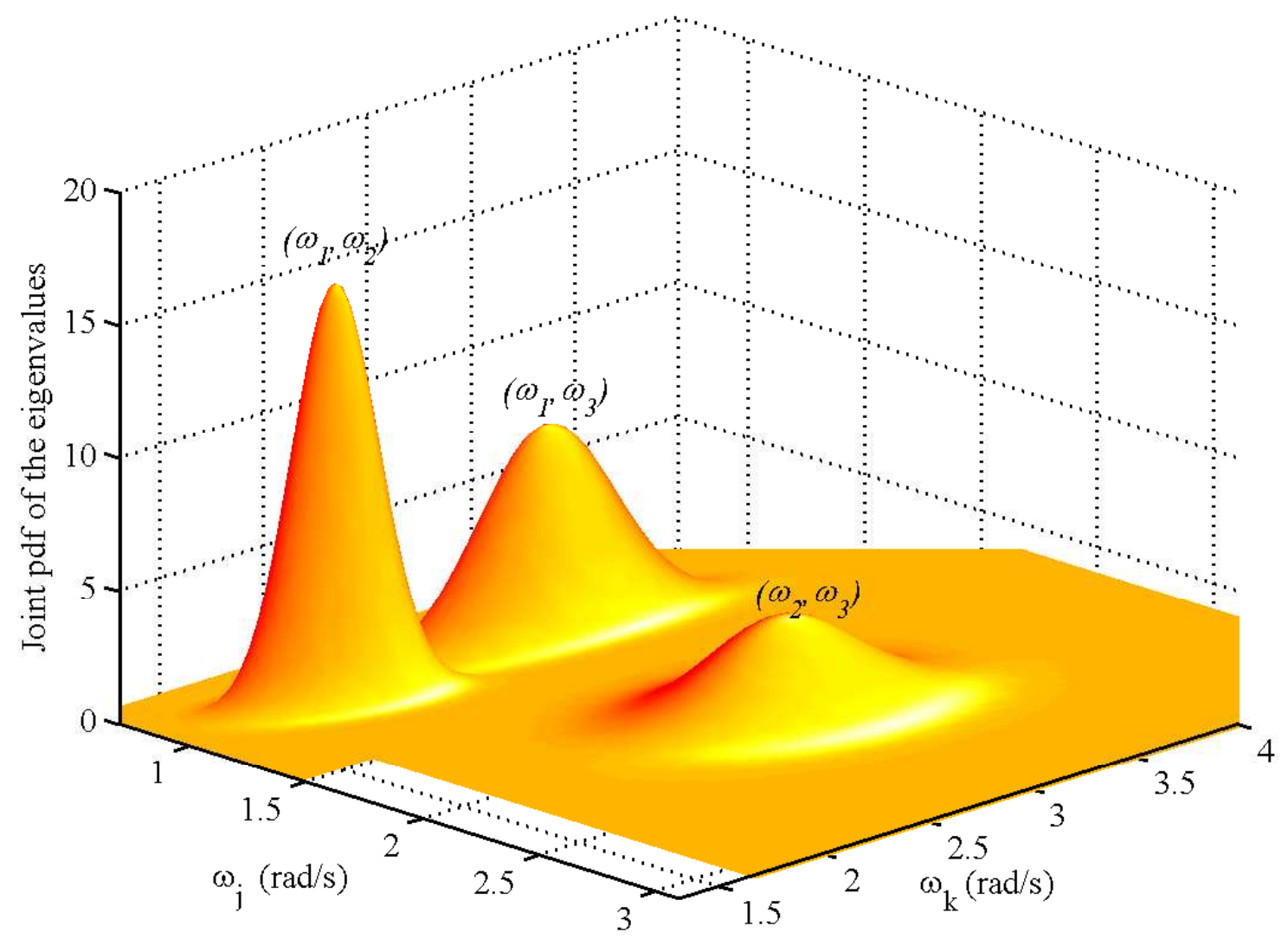

Figure 4. Fitted joint Gaussian probability density function of the natural frequencies using asymptotic method.

is, $\omega_{1}$ and $\omega_{2}$ and $\omega_{2}$ and $\omega_{3}$ are not jointly Gaussian distributed as the contours of the analytical joint pdf is quite different from that obtained using MCS. The joint pdf of $\omega_{1}$ and $\omega_{3}$ is however close to a bivariate Gaussian density function. The important conclusion that can be drawn from this limited numerical results is that the natural frequencies are in general not jointly Gaussian distributed although individually they may be. The exact expressions of the joint pdf of the natural frequencies derived in section II also support this conclusion for the special cases. Further research is however required to investigate the generality of this conclusion.

\section{Conclusions}

Joint statistics of the natural frequencies of discrete linear dynamic systems with parameter uncertainties have been considered. It is assumed that the mass and stiffness matrices are smooth and at least twice differentiable functions of a set of random variables. The random variables are in general considered to be non-Gaussian. The usual assumption of small randomness employed in most perturbation methods is not employed in this study. A general theorem on the exact evaluation of the joint probability density function of the natural frequencies of linear stochastic systems has been given. Based on this, some closed-form expressions of the joint probability density function of the natural frequencies have been reported for few special cases. Due to the lack of generality of the exact methods, an alternative approach based on asymptotic evaluation of multidimensional integrals has been suggested. A closed-form asymptotically correct expression for general order joint moments of arbitrary number of natural frequencies of linear stochastic systems with general non-Gaussian distribution has been derived. Using this expression, joint probability density function of the natural frequencies are calculated and compared with perturbation approximations and Monte Carlo Simulation for a three-degree-of-freedom random system. It was observed that the natural frequencies are 


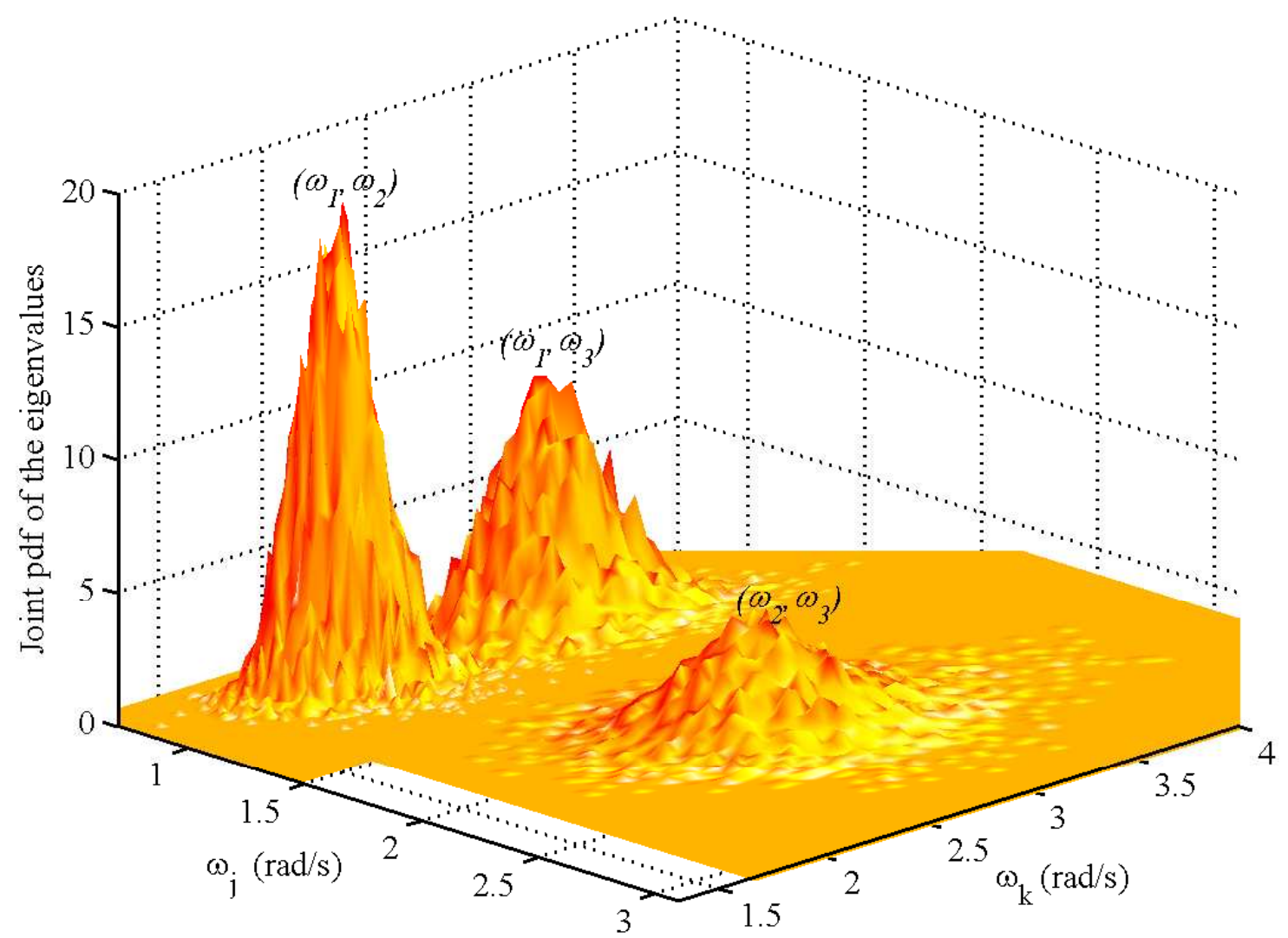

Figure 5. Joint probability density function of the natural frequencies from Monte Carlo Simulation.

not jointly Gaussian distributed although their marginal distribution are Gaussian. Generality of this result is however remains to be verified in future studies.

\section{A. Gradient Vector and Hessian Matrix of the Natural Frequencies}

The eigenvectors of symmetric linear systems are orthogonal with respect to the mass and stiffness matrices. Normalize the eigenvectors so that they are unity mass normalized, that is,

$$
\phi_{j}^{T} \mathbf{M} \phi_{j}=1
$$

Using this and differentiating Eq. (1) with respect to $x_{k}$ it can be shown that ${ }^{34}$ for any $\mathbf{x}$

$$
\begin{aligned}
\frac{\partial \omega_{j}(\mathbf{x})}{\partial x_{k}} & =\frac{\phi_{j}(\mathbf{x})^{T} \mathcal{G}_{j k}(\mathbf{x}) \phi_{j}(\mathbf{x})}{2 \omega_{j}(\mathbf{x})} \\
\text { where } \quad \mathcal{G}_{j k}(\mathbf{x}) & =\left[\frac{\partial \mathbf{K}(\mathbf{x})}{\partial x_{k}}-\omega_{j}^{2}(\mathbf{x}) \frac{\partial \mathbf{M}(\mathbf{x})}{\partial x_{k}}\right]
\end{aligned}
$$

Differentiating Eq. (1) with respect to $x_{k}$ and $x_{l}$ Plaut and Huseyin ${ }^{32}$ have shown that, providing the natural frequencies are distinct,

$$
\frac{\partial^{2} \omega_{j}(\mathbf{x})}{\partial x_{k} \partial x_{l}}=\left[\frac{1}{2 \omega_{j}(\mathbf{x})} \frac{\partial^{2}\left(\omega_{j}^{2}(\mathbf{x})\right)}{\partial x_{k} \partial x_{l}}-\frac{1}{\omega_{j}(\mathbf{x})} \frac{\partial \omega_{j}(\mathbf{x})}{\partial x_{l}} \frac{\partial \omega_{j}(\mathbf{x})}{\partial x_{k}}\right]
$$




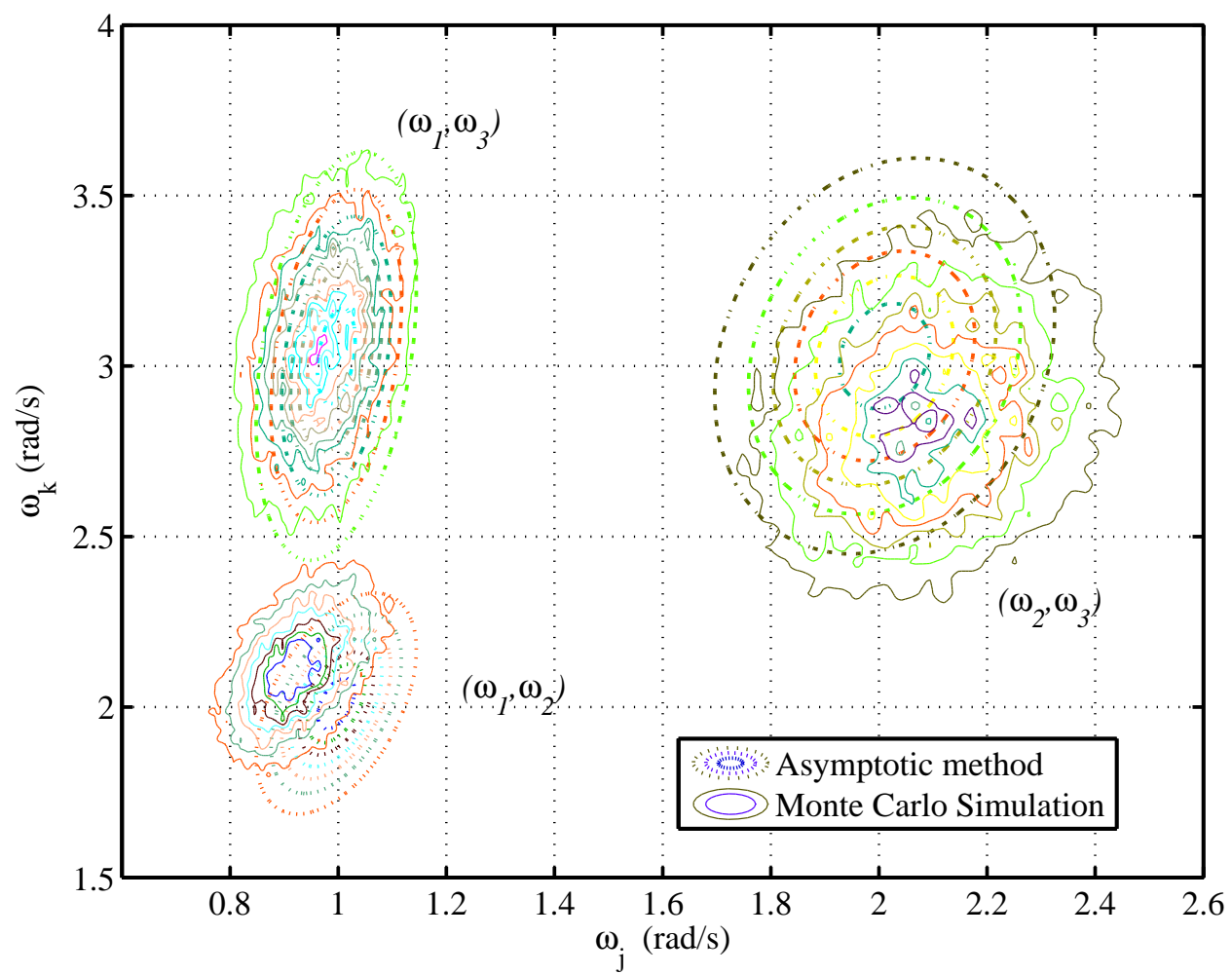

Figure 6. Contours of the joint probability density function of the natural frequencies.

where

$$
\begin{aligned}
& \frac{\partial^{2}\left(\omega_{j}^{2}(\mathbf{x})\right)}{\partial x_{k} \partial x_{l}}=\boldsymbol{\phi}_{j}(\mathbf{x})^{T}\left[\frac{\partial^{2} \mathbf{K}(\mathbf{x})}{\partial x_{k} \partial x_{l}}-\omega_{j}^{2}(\mathbf{x}) \frac{\partial^{2} \mathbf{M}(\mathbf{x})}{\partial x_{k} \partial x_{l}}\right] \boldsymbol{\phi}_{j}(\mathbf{x})-\left(\phi_{j}(\mathbf{x})^{T} \frac{\partial \mathbf{M}(\mathbf{x})}{\partial x_{k}} \boldsymbol{\phi}_{j}(\mathbf{x})\right)\left(\boldsymbol{\phi}_{j}(\mathbf{x})^{T} \mathcal{G}_{j l}(\mathbf{x}) \phi_{j}(\mathbf{x})\right) \\
& -\left(\boldsymbol{\phi}_{j}(\mathbf{x})^{T} \frac{\partial \mathbf{M}(\mathbf{x})}{\partial x_{l}} \boldsymbol{\phi}_{j}(\mathbf{x})\right)\left(\boldsymbol{\phi}_{j}(\mathbf{x})^{T} \mathcal{G}_{j k}(\mathbf{x}) \boldsymbol{\phi}_{j}(\mathbf{x})\right)+2 \sum_{r=1}^{N} \frac{\left(\boldsymbol{\phi}_{r}(\mathbf{x})^{T} \mathcal{G}_{j k}(\mathbf{x}) \boldsymbol{\phi}_{j}(\mathbf{x})\right)\left(\boldsymbol{\phi}_{r}(\mathbf{x})^{T} \mathcal{G}_{j l}(\mathbf{x}) \boldsymbol{\phi}_{j}(\mathbf{x})\right)}{\omega_{j}^{2}(\mathbf{x})-\omega_{r}^{2}(\mathbf{x})}
\end{aligned}
$$

Equations (77) and (79) completely define the elements of the gradient vector and Hessian matrix of the natural frequencies.

\section{B. Derivative of the System Matrices With Respect to the Random Variables}

The derivatives of $\mathbf{M}(\mathbf{x})$ and $\mathbf{K}(\mathbf{x})$ with respect to elements of $\mathbf{x}$ can be obtained from Eq. (62) together with Eqs. (63) and (64). For the mass matrix we have

$$
\frac{\partial \mathbf{M}}{\partial x_{1}}=\left[\begin{array}{ccc}
\bar{m}_{1} \epsilon_{m} & 0 & 0 \\
0 & 0 & 0 \\
0 & 0 & 0
\end{array}\right], \quad \frac{\partial \mathbf{M}}{\partial x_{2}}=\left[\begin{array}{ccc}
0 & 0 & 0 \\
0 & \bar{m}_{2} \epsilon_{m} & 0 \\
0 & 0 & 0
\end{array}\right], \quad \frac{\partial \mathbf{M}}{\partial x_{3}}=\left[\begin{array}{ccc}
0 & 0 & 0 \\
0 & 0 & 0 \\
0 & 0 & \bar{m}_{3} \epsilon_{m}
\end{array}\right]
$$


All other $\frac{\partial \mathbf{M}}{\partial x_{i}}$ are null matrices. For the derivative of the stiffness matrix

$$
\begin{aligned}
\frac{\partial \mathbf{K}}{\partial x_{4}} & =\left[\begin{array}{ccc}
\bar{k}_{1} \epsilon_{k} & 0 & 0 \\
0 & 0 & 0 \\
0 & 0 & 0
\end{array}\right], \quad \frac{\partial \mathbf{K}}{\partial x_{5}}=\left[\begin{array}{ccc}
0 & 0 & 0 \\
0 & \bar{k}_{2} \epsilon_{k} & 0 \\
0 & 0 & 0
\end{array}\right], \quad \frac{\partial \mathbf{M}}{\partial x_{6}}=\left[\begin{array}{ccc}
0 & 0 & 0 \\
0 & 0 & 0 \\
0 & 0 & \bar{k}_{3} \epsilon_{k}
\end{array}\right] \\
\frac{\partial \mathbf{K}}{\partial x_{7}} & =\left[\begin{array}{ccc}
\bar{k}_{4} \epsilon_{k} & -\bar{k}_{4} \epsilon_{k} & 0 \\
-\bar{k}_{4} \epsilon_{k} & \bar{k}_{4} \epsilon_{k} & 0 \\
0 & 0 & 0
\end{array}\right], \quad \frac{\partial \mathbf{K}}{\partial x_{8}}=\left[\begin{array}{ccc}
0 & 0 & 0 \\
0 & \bar{k}_{5} \epsilon_{k} & -\bar{k}_{5} \epsilon_{k} \\
0 & -\bar{k}_{5} \epsilon_{k} & \bar{k}_{5} \epsilon_{k}
\end{array}\right], \quad \frac{\partial \mathbf{M}}{\partial x_{9}}=\left[\begin{array}{ccc}
\bar{k}_{6} \epsilon_{k} & 0 & -\bar{k}_{6} \epsilon_{k} \\
0 & 0 & 0 \\
-\bar{k}_{6} \epsilon_{k} & 0 & \bar{k}_{6} \epsilon_{k}
\end{array}\right]
\end{aligned}
$$

and all other $\frac{\partial \mathbf{K}}{\partial x_{i}}$ are null matrices. Also note that all of the first-order derivative matrices are independent of $\mathbf{x}$. For this reason, all the higher order derivatives of the $\mathbf{M}(\mathbf{x})$ and $\mathbf{K}(\mathbf{x})$ matrices are null matrices.

\section{Acknowledgments}

The author acknowledges the support of the UK Engineering and Physical Sciences Research Council (EPSRC) through the award of an Advanced Research Fellowship, grant number GR/T03369/01. The author is grateful to Prof. M. I. Friswell for numerous helpful discussions.

\section{References} 1968.

${ }^{1}$ Boyce, W. E., Random Eigenvalue Problems, Probabilistic methods in applied mathematics, Academic Press, New York,

${ }^{2}$ Scheidt, J. V. and Purkert, W., Random Eigenvalue Problems, North Holland, New York, 1983.

${ }^{3}$ Ibrahim, R. A., "Structural dynamics with parameter uncertainties," Applied Mechanics Reviews, ASME, Vol. 40, No. 3, 1987, pp. 309-328.

${ }^{4}$ Benaroya, H. and Rehak, M., "Finite element methods in probabilistic structural analysis: a selective review," Applied Mechanics Reviews, ASME, Vol. 41, No. 5, 1988, pp. 201-213.

${ }^{5}$ Benaroya, H., "Random eigenvalues, algebraic methods and structural dynamic models," Applied Mathematics and Computation, Vol. 52, 1992, pp. 37-66.

${ }^{6}$ Manohar, C. S. and Ibrahim, R. A., "Progress in structural dynamics with stochastic parameter variations: 1987 to 1998," Applied Mechanics Reviews, ASME, Vol. 52, No. 5, May 1999, pp. 177-197.

${ }^{7}$ Manohar, C. S. and Gupta, S., "Modeling and evaluation of structural reliability: Current status and future directions," Research reviews in structural engineering, Golden Jubilee Publications of Department of Civil Engineering, Indian Institute of Science, Bangalore, edited by K. S. Jagadish and R. N. Iyengar, University Press, 2003.

${ }^{8}$ Grigoriu, M., "A solution of random eigenvalue problem by crossing theory," Journal of Sound and Vibration, Vol. 158, No. 1, 1992, pp. 69-80.

${ }^{9}$ Lee, C. and Singh, R., "Analysis of discrete vibratory systems with parameter uncertainties, part I: Eigensolution," Journal of Sound and Vibration, Vol. 174, No. 3, 1994, pp. 379-394.

${ }^{10}$ Nair, P. B. and Keane, A. J., "An approximate solution scheme for the algebraic random eigenvalue problem," Journal of Sound and Vibration, Vol. 260, No. 1, 2003, pp. 45-65.

${ }^{11}$ Adhikari, S. and Langley, R. S., "Distribution of eigenvalues of linear stochastic systems," Proceedings of the ninth International Conference on Applications of Statistics and Probability in Civil Engineering (ICASP 9), San Fransisco, California, USA, edited by A. Der-Kiureghian, S. Madanat, and J. M. Pestana, Vol. 1 of Applications of Statistics and Probability in Civil Engineering, Millpress, Rotterdam, Netherlands, July 2003, pp. 201-207.

${ }^{12}$ Adhikari, S. and Friswell, M. I., "Random eigenvalue problems in structural dynamics," 45th AIAA/ASME/ASCE Structures, Structural Dynamics \&s Materials Conference, Palm Springs, CA, 2004.

${ }^{13}$ Mehta, M. L., Random Matrices, Academic Press, San Diego, CA, 2nd ed., 1991.

${ }^{14}$ Collins, J. D. and Thomson, W. T., "The eigenvalue problem for structural systems with statistical properties," AIAA Journal, Vol. 7, No. 4, April 1969, pp. 642-648.

${ }^{15}$ Muirhead, R. J., Aspects of Multivariate Statistical Theory, John Wiely and Sons, New York, USA, 1982.

${ }^{16}$ Brody, T. A., Flores, J., French, J. B., Mello, P. A., Pandey, A., and Wong, S. S. M., "Random matrix physics: spectrum and strength fluctuations." Reviews of Modern Physics, Vol. 53, No. 3, 1981, pp. 385-480.

${ }^{17}$ Langley, R. S. and Brown, A. W. M., "The ensemble statistics of the energy of a random system subjected to harmonic excitation," Journal of Sound and Vibration, 2003, To appear.

${ }^{18}$ Langley, R. S. and Brown, A. W. M., "The ensemble statistics of the band-averaged energy of a random system," Journal of Sound and Vibration, 2003, To appear.

${ }^{19}$ Langley, R. S. and Brown, A. W. M., "Numerical evidence for the Wigner surmise," Journal of the Acoustical Society of America, Vol. 109, 2001, pp. 2443

${ }^{20}$ Gupta, A. and Nagar, D., Matrix Variate Distributions, Monographs \& Surveys in Pure \& Applied Mathematics, Chapman \& Hall/CRC, London, 2000. 
${ }^{21}$ Dumitriu, I. and Edelman, A., "Matrix models for beta ensembles," Journal of Mathematical Physics, Vol. 43, 2002, pp. $5830-5847$.

${ }^{22}$ Hasselman, T. K. and Hart, G. C., "Modal analysis of random structural system," Journal of Engineering Mechanics, ASCE, Vol. 98, No. EM3, June 1972, pp. 561-579.

${ }^{23}$ Hart, G. C., "Eigenvalue uncertainties in stressed structure," Journal of Engineering Mechanics, ASCE, Vol. 99, No. EM3, June 1973, pp. 481-494.

${ }^{24}$ Ramu, S. A. and Ganesan, R., "A Galerkin finite element technique for stochastic field problems," Computer Methods in Applied Mechanics andEngineering, Vol. 105, 1993, pp. 315-331.

${ }^{25}$ Sankar, T. S., Ramu, S. A., and Ganesan, R., "Stochastic finite element analysis for high speed rotors," Journal of Vibration and Accoustic, ASME, Vol. 115, 1993, pp. 59-64.

${ }^{26}$ Adhikari, S., "Complex Modes in Stochastic Systems," Advances in Vibration Engineering, Vol. 3, No. 1, 2004, pp. 1-11.

${ }^{27}$ Johnson, N. L. and Kotz, S., Distributions in Statistics: Continuous Univariate Distributions - 2, The Houghton Mifflin Series in Statistics, Houghton Mifflin Company, Boston, USA, 1970.

${ }^{28}$ Mathai, A. M. and Provost, S. B., Quadratic Forms in Random Variables: Theory and Applications, Marcel Dekker, Inc., 270 Madison Avenue, New York, NY 10016, USA, 1992.

${ }^{29}$ Bleistein, N. and Handelsman, R. A., Asymptotic Expansions of Integrals, Holt, Rinehart and Winston, New York, 1994.

${ }^{30}$ Wong, R., Asymptotic Approximations of Integrals, Society of Industrial and Applied Mathematics, Philadelphia, PA, USA, 2001, First published by Academic Press Inc. in 1989.

${ }^{31}$ Papadimitriou, C., Beck, J. L., and Katafygiotis, L. S., "Asymptotic expansions for reliability and moments of uncertain systems," Journal of Engineering Mechanics, ASCE, Vol. 123, No. 12, December 1997, pp. 1219-1229.

${ }^{32}$ Plaut, R. H. and Huseyin, K., "Derivative of eigenvalues and eigenvectors in non-self adjoint systems," AIAA Journal, Vol. 11, No. 2, February 1973, pp. 250-251.

${ }^{33}$ Friswell, M. I., "The derivatives of repeated eigenvalues and their associated eigenvectors," ASME Journal of Vibration and Acoustics, Vol. 18, July 1996, pp. 390-397.

${ }^{34}$ Fox, R. L. and Kapoor, M. P., "Rates of change of eigenvalues and eigenvectors," AIAA Journal, Vol. 6, No. 12, December 1968, pp. 2426-2429.

\section{Nomenclature}

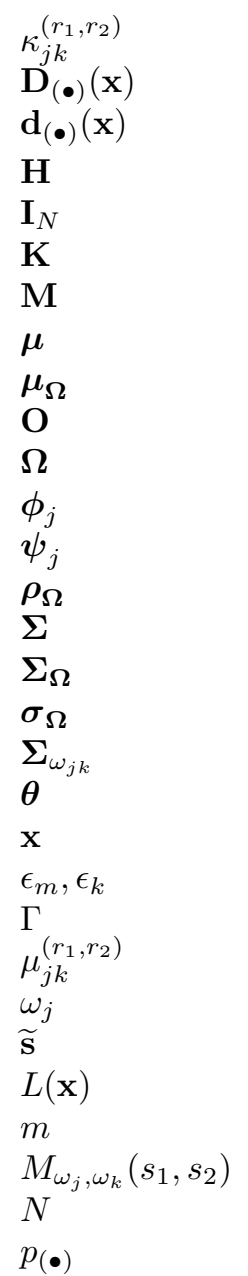

$\left(r_{1}, r_{2}\right)$-th order cumulant of $j$ th and $k$ th natural frequencies

Hessian matrix of $(\bullet)$ at $\mathbf{x}$

gradient vector of $(\bullet)$ at $\mathbf{x}$

symmetric system matrix, $\mathbf{H}=\mathbf{M}^{-1 / 2} \mathbf{K M}^{-1 / 2}$

identity matrix of size $N$

stiffness matrix

mass matrix

mean of parameter vector $\mathbf{x}$

mean of the natural frequencies

null matrix

diagonal matrix containing the natural frequencies

$j$ th mode shape of the system

eigenvectors of the symmetric system matrix $\mathbf{H}$

correlation coefficient matrix of the natural frequencies

covariance matrix of parameter vectors $\mathbf{x}$

covariance matrix of the natural frequencies

standard deviation of the natural frequencies

covariance matrix of $j$ th and $k$ th natural frequencies

optimal point for asymptotic method

basic random variables

strength parameters associated with mass and stiffness coefficients

$\Gamma$ function

$\left(r_{1}, r_{2}\right)$-th order joint moment of $j$ th and $k$ th natural frequencies

$j$ th natural frequencies of the system

vector of complex Laplace parameters $s_{1}$ and $s_{2}$

negative of the log-likelihood function

number of basic random variables

joint moment generating function of $\omega_{j}$ and $\omega_{k}$

degrees-of-freedom of the system

probability density function of $(\bullet)$ 


\begin{tabular}{ll}
$(\bullet)^{T}$ & matrix transpose \\
$\approx$ & approximately equal to \\
$\mathbb{C}$ & space of complex numbers \\
$\mathbb{R}$ & space of real numbers \\
$\mathrm{Cov}(\bullet, \bullet)$ & covariance of random quantities \\
$\mathrm{Cov}(\bullet, \bullet)$ & covariance of random quantities \\
$\|\bullet\|$ & determinant of a matrix \\
$\exp$ & exponential function \\
$\mathrm{E}[\bullet]$ & expectation operator \\
$\in$ & belongs to \\
$\mapsto$ & maps into \\
$|\bullet|$ & $l_{2}$ norm of $(\bullet)$ \\
$\otimes$ & Kronecker product \\
\hline$(\bullet)$ & deterministic value of $(\bullet)$ \\
$O(N)$ & group of $N \times N$ orthogonal matrices \\
dof & degrees-of-freedom \\
jpdf & joint probability density function \\
pdf & probability density function
\end{tabular}

